\title{
A New Concept of the Stock-Recruitment Relationship for the Japanese Sardine, Sardinops Melanostictus
}

\author{
Kazumi Sakuramoto*
}

Tokyo University of Marine Science and Technology, 4-5-7 Konan, Minato-ku, Tokyo 108-8477, Japan

\begin{abstract}
The aim of this paper is to discuss the validity of the results of Wada and Jacobson (Can. J. Fish. Aquat. Sci. $55,1998)$ and to propose a new concept of the stock-recruitment relationship for the Japanese sardine. Two regression analyses were used: simple regression and Deming regression. Reproductive success was defined as recruitment (R) divided by egg production. A false decreasing trend was produced in the regression line of $\ln$ (reproductive success) against $\ln$ (egg production) in response to observation error. When Deming regression was applied, the slope of the regression line of $\ln (\mathrm{R})$ against $\ln$ (egg production) did not statistically differ from unity. This indicates that a density-dependent effect cannot be detected on the stock-recruitment relationship in the data used in this study and that it is reasonable instead to accept a proportional model as the optimal stock-recruitment relationship model for the Japanese sardine. The results of this study are diametrically opposed to those of Wada and Jacobson (1998), who assumed that density-dependent effects on recruitment typically exist for Sardinops species.
\end{abstract}

Keywords: Density-dependent effect, MSY, recruitment, Ricker, regime shift, reproductive success, sardine, stock-recruitment relationship.

\section{INTRODUCTION}

The need to understand the mechanisms underlying fluctuations in fish population size is readily apparent. Densitydependent effects intrinsic to the population are one of the main factors thought to cause populations to fluctuate. The existence of density-dependent effects is suggested by both recruitment $(\mathrm{R})$ versus egg production and reproductive success (recruitments per egg production) versus egg production relationships. If density-dependent effects exist, they respectively produce domed [1] or asymptotic [2] curves in plots of $\mathrm{R}$ against egg production, and decreasing slopes in plots of reproductive success against egg production.

Wada and Jacobson [3] examined reproductive success and annual egg production relationship for sardines (Sardinops spp.) and discussed how and why they fluctuated, invoking the concept of the "regime shift." They only used reproductive success as the dependent variable of the regression analysis because reproductive success in sardines responds rapidly to environmental changes [3]. They separated their multi-year data into periods or "regimes" which they designated as "favorable" and "unfavorable", and calculated the regression lines of $\ln$ (reproductive success) against $\ln$ (egg production) for each regime. From data for the Japanese sardine and the Pacific sardine off the coast of California [4], they first concluded that density-dependent effects on recruitment do indeed typically exist for Sardinops species, but that they can only be measured using long-term data

*Address correspondence to this author at the Tokyo University of Marine Science and Technology, 4-5-7 Konan, Minato-ku, Tokyo 108-8477, Japan; Tel: 81-3-5463-0563; Fax: 81-3-5463-0563,

E-mail: sakurak@kaiyodai.ac.jp series exhibiting large (1000-fold) changes in abundance [5]. Second, they determined that intercept parameters from the spawner-recruitment models could be used to test hypotheses concerning regime differences, and that slope parameters could be used to test hypotheses concerning densitydependent effects. Third, they concluded that densitydependent effects of sardine egg production on reproductive success were similar under the two different environmental regimes. Fourth, their regression parameter estimates allowed them to propose that the carrying capacity for the Japanese sardine during the favorable regime of 1971-1987 was about 75 times greater than the carrying capacities during the unfavorable regimes of 1951-1970 and 1988-1991.

Wada and Jacobson's [3] work has been cited in many papers [6-10], and is truly a key paper in the analysis of Sardinops population dynamics. However, in the present paper, I take to task several of Wada and Jacobson's [3] conclusions on various grounds. First, it is unclear what evidence they used to base their assertion that reproductive success respond rapidly to environmental changes upon. Second, it is unclear why they did not exclude from analysis their 19881991 data, four years that are considered anomalous because levels of reproductive success were extraordinarily low in an apparent response to marine environmental conditions [11]. Third, it is unclear why Wada and Jacobson [3] did exclude their 1992-1995 data: they claim that they were not confident in the accuracy of the value estimates for 1992-1995 [3], but their rationale for excluding the last four years of the data series (rather than the last three or five years) is uncertain. Fourth, it has not been objectively verified that the environmental conditions pertaining to this time series can be validly separated into two distinct regimes (favorable and unfavorable), as Wada and Jacobson [3] contend. 
Table 1. Artificial Data for the Simulations. Nine Pairs of Egg Production $(E)$ and Recruitment $(R)$ data are assumed. In Simulation 1, the true $E$ and $R$ are both, for simplicity, same at 500, however, they are underestimated by 0.5 -fold and overestimated by 2.0 -fold, respectively. Then the following nine observed pairs $(E, R)$ and reproductive success $(E, R P S)$ are generated. The second simulation assumes a different regime in which $E$ and $R$ are both 10 times larger than the case in Simulation 1

\begin{tabular}{|c|c|c|c|c|c|c|c|}
\hline \multicolumn{9}{|c|}{ Simulation 1 } & \multicolumn{4}{|c|}{ Simulation 2 } \\
\hline $\begin{array}{c}\text { True } \\
(\boldsymbol{E}, \boldsymbol{R})\end{array}$ & $\begin{array}{c}\text { True } \\
\boldsymbol{R} \boldsymbol{S}\end{array}$ & $\begin{array}{c}\text { Observed } \\
(\boldsymbol{E}, \boldsymbol{R})\end{array}$ & $\begin{array}{c}\text { Observed } \\
(\boldsymbol{E}, \boldsymbol{R} \boldsymbol{P S})\end{array}$ & $\begin{array}{c}\text { True } \\
(\boldsymbol{E}, \boldsymbol{R})\end{array}$ & $\begin{array}{c}\text { True } \\
\boldsymbol{R} \boldsymbol{S} \boldsymbol{S}\end{array}$ & $\begin{array}{c}\text { Observed } \\
(\boldsymbol{E}, \boldsymbol{R})\end{array}$ & $\begin{array}{c}\text { Observed } \\
(\boldsymbol{E}, \boldsymbol{R} \boldsymbol{P} \boldsymbol{)})\end{array}$ \\
\hline \hline$(500,500)$ & 1.00 & $(250,250)$ & $(250,1.00)$ & $(5000,5000)$ & 1.00 & $(2500,2500)$ & $(2500,1.00)$ \\
\hline$(500,500)$ & 1.00 & $(250,500)$ & $(250,2.00)$ & $(5000,5000)$ & 1.00 & $(2500,5000)$ & $(2500,2.00)$ \\
\hline$(500,500)$ & 1.00 & $(250,1000)$ & $(250,4.00)$ & $(5000,5000)$ & 1.00 & $(2500,10000)$ & $(2500,4.00)$ \\
\hline$(500,500)$ & 1.00 & $(500,250)$ & $(500,0.50)$ & $(5000,5000)$ & 1.00 & $(5000,2500)$ & $(5000,0.50)$ \\
\hline$(500,500)$ & 1.00 & $(500,500)$ & $(500,1.00)$ & $(5000,5000)$ & 1.00 & $(5000,5000)$ & $(5000,1.00)$ \\
\hline$(500,500)$ & 1.00 & $(500,1000)$ & $(500,2.00)$ & $(5000,5000)$ & 1.00 & $(5000,10000)$ & $(5000,2.00)$ \\
\hline$(500,500)$ & 1.00 & $(1000,250)$ & $(1000,0.25)$ & $(5000,5000)$ & 1.00 & $(10000,2500)$ & $(10000,0.25)$ \\
\hline$(500,500)$ & 1.00 & $(1000,500)$ & $(1000,0.50)$ & $(5000,5000)$ & 1.00 & $(10000,5000)$ & $(10000,0.50)$ \\
\hline$(500,500)$ & 1.00 & $(1000,1000)$ & $(1000,1.00)$ & $(5000,5000)$ & 1.00 & $(10000,10000)$ & $(10000,1.00)$ \\
\hline
\end{tabular}

Table 2. Results of the regression analysis for the case when (1) $\ln (\mathrm{RPS})=\mathbf{a}+\mathbf{b} \cdot \ln (\operatorname{egg}$ production $)$ or $(2) \ln (\mathrm{R})=\mathbf{a}+\mathbf{b} \cdot \ln (\mathrm{egg}$ production). RPS denotes the reproductive success. The first and second rows show the estimated values by Wada and Jacobson [3]. In both periods (favorable and unfavorable regimes), they assumed the same slope $b$ and estimated the slope at $\mathbf{- 0 . 7 0 1}$. "Simple" in the first column indicates the simple regression analysis. Column 5-8 shows the estimated slopes, their $\mathbf{9 5 \%}$ confidence limits and significant probability (p) value of the slope of the line. The last column indicates whether or not the estimated slope is recognized statistically differ from zero for model (1) or unity for model (2), respectively. If the slope is statistically differ from zero or unity, it indicates that the density-dependent effect is not detected. Bottom row shows the results when the deming regression was applied for all period of data from 1951-1991.

\begin{tabular}{|c|c|c|c|c|c|c|c|c|}
\hline \multirow[t]{2}{*}{ Method } & \multirow[t]{2}{*}{ Data Year Span } & \multirow{2}{*}{$\begin{array}{c}\text { Regression } \\
\text { Model }\end{array}$} & \multirow[t]{2}{*}{$\begin{array}{c}\text { Figure } \\
\text { Displayed }\end{array}$} & \multirow[t]{2}{*}{$b$} & \multicolumn{2}{|c|}{$\begin{array}{l}\text { 95\% Confidence Limit } \\
\text { for Slope Value }\end{array}$} & \multirow{2}{*}{$\begin{array}{l}p \text { Value for } \\
\text { Slope Value }\end{array}$} & \multirow{2}{*}{$\begin{array}{l}\text { Evaluation } \\
\text { of Slope } b\end{array}$} \\
\hline & & & & & Lower & Higher & & \\
\hline W-J & 1971-1987 & (1) & Fig. (2a) & -0.701 & --- & --- & --- & $\mathrm{b}<0$ \\
\hline W-J & $1951-1970,1988-1991$ & (1) & Fig. (2a) & -0.701 & --- & --- & --- & $\mathrm{b}<0$ \\
\hline Simple & 1951-1991 & (1) & Fig. (2b) & -0.229 & -0.423 & -0.035 & 0.022 & $\mathrm{~b}<0$ \\
\hline Simple & 1951-1987 & (1) & Fig. (2c) & -0.021 & -0.199 & 0.157 & 0.812 & $\mathrm{~b}=0$ \\
\hline Simple & 1951-1972 & (1) & Fig. $(\mathbf{3 b}, \mathbf{c})$ & -0.902 & -1.552 & -0.252 & 0.009 & $\mathrm{~b}<0$ \\
\hline Simple & 1974-1995 & (1) & Fig. (3b) & -0.718 & -1.191 & -0.246 & 0.005 & $\mathrm{~b}<0$ \\
\hline Simple & 1951-1995 & (1) & Fig. (3b) & -0.231 & -0.415 & -0.047 & 0.015 & $\mathrm{~b}<0$ \\
\hline Simple & $1974-1987,1992-1995$ & (1) & Fig. (3c) & -0.236 & -0.489 & 0.018 & 0.067 & $\mathrm{~b}<0^{* 1}$ \\
\hline Simple & 1951-1987, 1992-1995 & (1) & Fig. (3c) & -0.036 & -0.204 & 0.132 & 0.665 & $\mathrm{~b}=0$ \\
\hline Simple & Simulation 1 or 2 & (1) & Fig. (4) & -1.000 & -1.894 & -0.106 & 0.033 & $\mathrm{~b}<0$ \\
\hline Simple & Simulation 1 and 2 & (1) & Fig. (4) & -0.195 & -0.509 & 0.120 & 0.208 & $\mathrm{~b}=0$ \\
\hline Simple & 1951-1991 & (2) & Fig. (5) & 0.771 & 0.577 & 0.965 & $8.59\left(10^{-10}\right)$ & $\mathrm{b}<1$ \\
\hline Simple & $1951-1987$ & (2) & Fig. (5) & 0.979 & 0.801 & 1.157 & $4.50\left(10^{-13}\right)$ & $\mathrm{b}=1$ \\
\hline Deming & 1951-1991 & (2) & Fig. (5) & 0.971 & 0.866 & 1.099 & --- & $\mathrm{b}=1$ \\
\hline
\end{tabular}

*1 This slope is also statistically negative under the $10 \%$ significant level.

The aim of this paper is to examine the preceding issues, the goal being to better understand the nature of Japanese sardine population fluctuations and the mechanisms underlying them, in hopes of managing these populations on the soundest bases possible.

\section{MATERIALS AND METHODOLOGY}

I base my results on re-analyses of the recruitment $(\mathrm{R})$ and annual egg production data (1951-1996) from Table 2 of Wada and Jacobson [3]. I address two relationships: the regression of $\ln$ (reproductive success) against $\ln$ (egg production) and the regression of $\ln (\mathrm{R})$ against $\ln (\mathrm{egg}$ production). Two kinds of regression analyses were applied to these data sets.

The first regression approach I applied to the data was the simple, traditionally-used, least-squares regression. However, this approach is problematic in that it assumes no observation error in the independent variable. Stöckl et al. [12] noted that when observation error in the predictor variable does occur, the parameter estimates derived from least- 

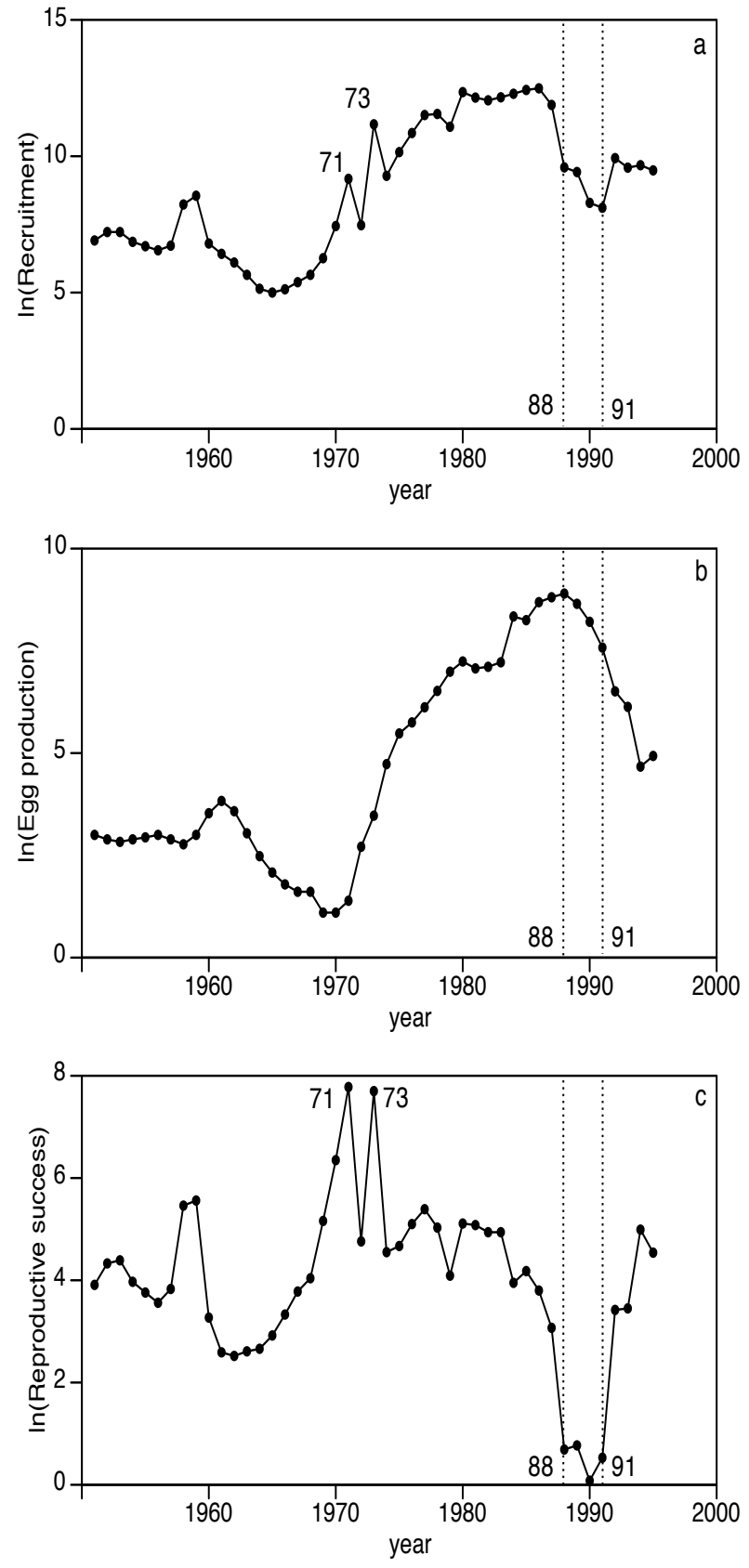

Fig. (1). Fluctuations of (a) $\ln ($ recruitment), (b) $\ln ($ egg production), and (c) $\ln$ (reproductive success) through time (1951-1995) for Japanese sardines. Data from Wada and Jacobson [3]. Vertical dotted lines indicates the year from 1988-1991 when the anomalous low reproductive success occurred. In 1971 and 1973, the reproductive success was also extremely high. It would occur in response to the huge recruitment in 1971 and 1973.

squared regression exhibit bias; thus, the use of simple regression in such situations has recognized drawbacks.

The other regression approach I applied to the data was Deming regression [13]. For this method, observation errors are assumed to occur in both the dependent and independent variables. The algorithm for Deming regression was developed by Aoki [14]. For the Deming regression analyses that I conducted, the ratios of the variances in $\mathrm{R}$ and egg produc- tion were assumed to be 1 . Other ratios were also used as the sensitivity tests.

In order to reproduce the graph that is obtained when we draw the graph of $\ln$ (reproductive success) against $\ln (\mathrm{egg}$ production) for all the data from 1951 -1995 (shown later), and in order to test the validity of the assertion that reproductive success respond rapidly to environmental changes and the validity of drawing the two regression lines for the relationship between $\ln$ (reproductive success) against $\ln (\mathrm{egg}$ production) for favorable and unfavorable regimes, I conducted two simple and deterministic numerical simulations. In simulation 1 , we consider the case when nine sets of recruitment, $R$ and egg production, $E$, i.e., $\left(E_{i}, R_{i}\right),(i=1,2$, ...9) are observed. Further, for simplicity, we consider the case when all the true $R_{i}(i=1,2, \ldots 9)$ and $E_{i}$ values $(i=1$, $2, \ldots 9)$ are same at 500 , respectively. Then all the true reproductive success $\left(R P S_{i}\right)$ calculated by $R_{i} / E_{i}$ are unity. We assume only two kinds of observation errors occurs, i.e., 0.5 fold underestimation or 2.0-fold overestimation. Therefore, resultant observation values are 250 (underestimation), 500 (no observation error) or 1000 (overestimation) for both $\mathrm{R}$ and egg production, respectively. Then we can obtain the nine pairs of egg production and reproductive success $\left(E_{i}\right.$, $\left.R P S_{i}\right),(i=1,2, \ldots 9)$ shown in Table 1.

In simulation 2 , I assumed a regime shift and the true values of $\mathrm{R}$ and egg production were both, for instance, 10fold greater than the simulation 1 (Table 1). In simulation 2, the observation errors occur in a same manner as in simulation 1 , i.e. $R$ and egg production are underestimated by 0.5 fold or overestimated by 2.0 -fold (Table 1). Therefore, in simulation 2 , we can obtain nine pairs of $\left(E_{i}, R_{i}\right),(i=1,2$, $\ldots 9)$ and $\left(E_{i}, R P S_{i}\right),(i=1,2, \ldots 9)$ under the same manner of simulation 1 .

\section{RESULTS}

\section{Temporal Variation}

Fig. (1a, b, and c), respectively, show the fluctuations in $\ln (\mathrm{R}), \ln ($ egg production), and $\ln$ (reproductive successes) over time (1951-1995).

The $\ln (\mathrm{R})$ values increased into the mid-1960s, plateau during the1980s, but decreased from 1988 through 1991, after which it recovered in 1992 to levels typical of the early 1970s.

The $\ln$ (egg production) values largely varied with time as did the $\ln (\mathrm{R})$ values. Increases beginning in the early 1970 s likely were in response to the increase in recruitment. A maximum was reached in 1988, after which a decrease began in 1989, likely in response to extremely low levels of recruitment.

The declines in $\ln ($ egg production) paralleled those in $\ln (\mathrm{R})$. However, the reduction in $\ln ($ egg production) lagged behind by two years relative to $\ln (\mathrm{R})$, and the steepness of the reduction in $\ln$ (egg production) was not as severe as that in $\ln (\mathrm{R})$, likely because the spawners were made up of mainly two-year-olds and older. The effect of the decrease in recruitment on egg production was not large.

In contrast, the variation in $\ln$ (reproductive successes) values over time strongly reflected the decrease in recruitment. This is clearly shown in Fig (1c). The $\ln$ (reproductive 

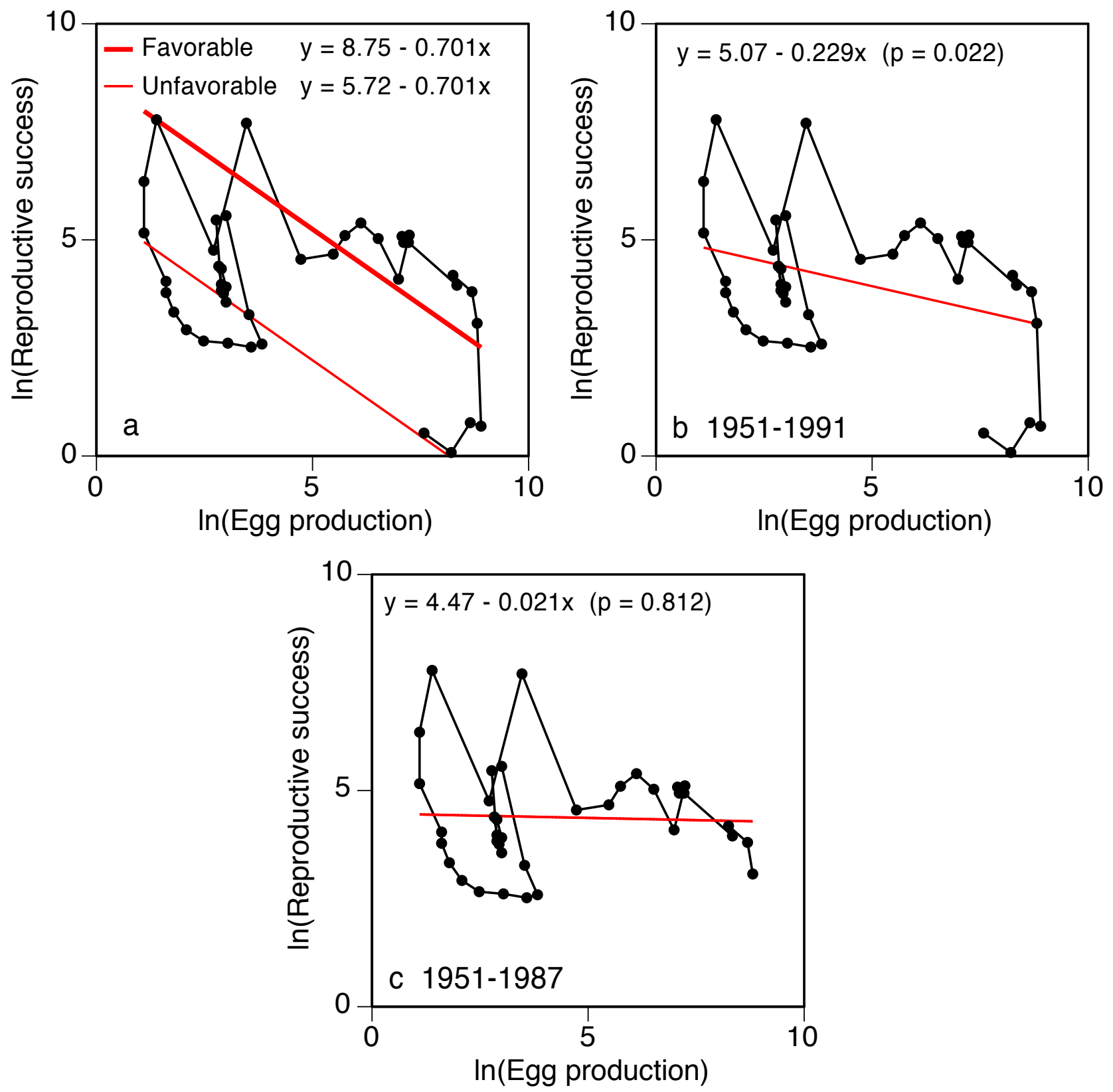

Fig. (2). Relationship between $\ln$ (reproductive success) and $\ln$ (egg production). (a) Reproduction of Fig. 3 from Wada and Jacobson [3], Thick and thin regression lines indicate the regression lines adapted for favorable and unfavorable regimes, separately. (b) one regression line adapted for all the data from 1951 to 1991 . The slope is -0.229 and significantly negative ( $p=0.022)$, i.e., density-dependent effect is detected. Here, $x$ and $y$ denote the $\ln ($ egg production) and $\ln$ (reproductive success), respectively. (c) regression line for the data when the anomalous four years from 1988 to 1991 are removed. The slope is -0.021 and not significantly different from zero $(p=0.812)$. This indicates that density-dependent effect is not detected when the anomalous four years are removed.

successes) values in 1988-1991 were extremely low compared to the other years, whereas the $\ln$ (reproductive successes) values for 1971 and 1973 were high in apparent response to the high $\ln (\mathrm{R})$ values for these years.

\section{Results with 1988-1991 Data Excluded}

Fig. (2a) reproduces Fig. (3) from Wada and Jacobson [3]. At first glance, the two regression lines appear to accurately describe the relationships between $\ln$ (reproductive successes) and $\ln$ (egg production) for both the favorable and unfavorable environmental regimes.
Fig. (2b) shows the same plots, but without the two regression lines point. Instead, a single regression line for all the points is depicted, the slope of which is both negative ($0.229)$ and significantly different from zero $(p=0.022)$. However, note that the values for the years 1988-1991 are markedly low.

With these points excluded, the resulting regression line almost parallels the $\mathrm{X}$ axis (Fig. 2c), having a slope of -0.021 that does not differ significantly from zero $(p=0.812)$. As was mentioned previously, these four years were characterized by anomalous environmental conditions [11]. Including 

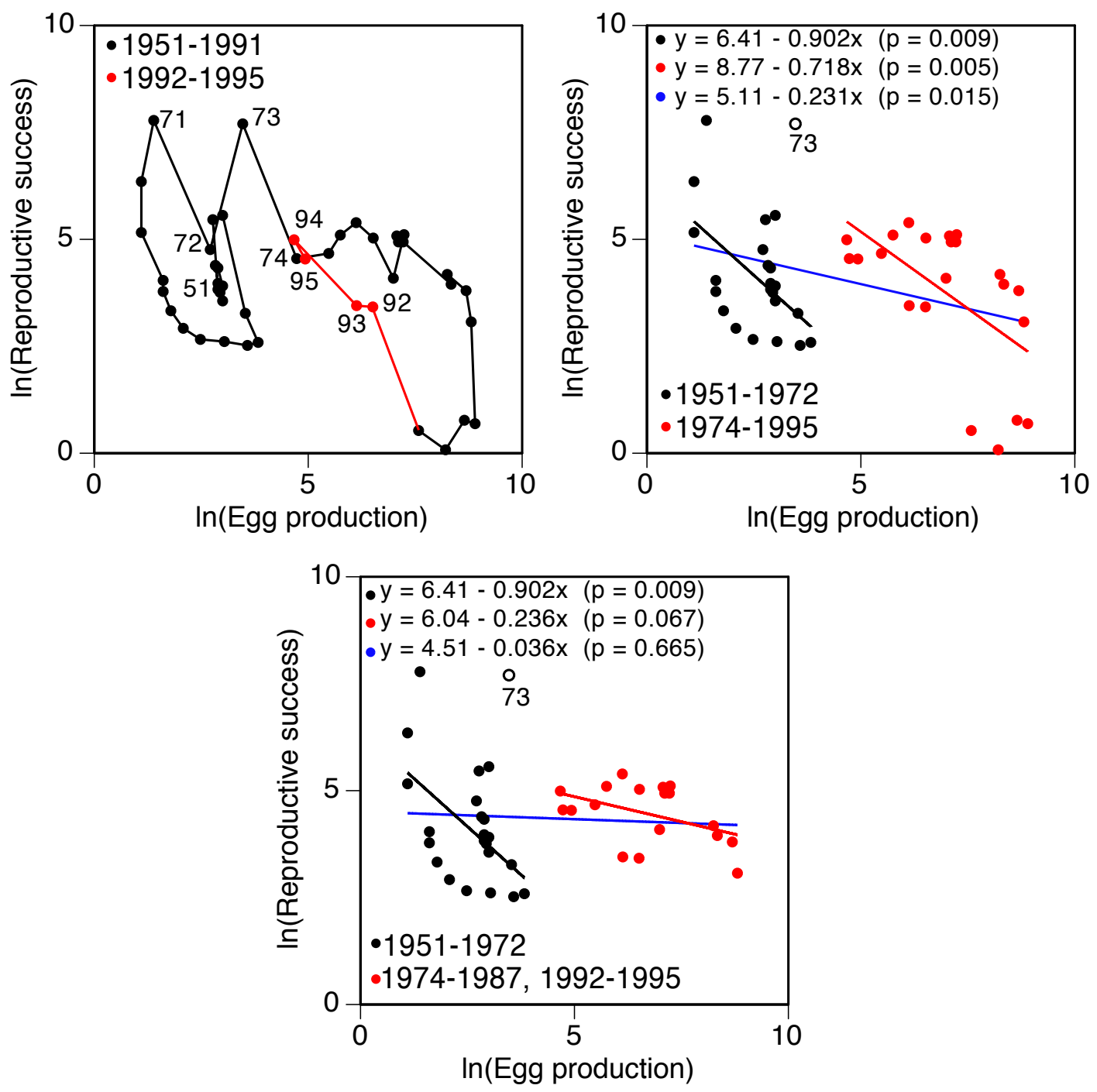

Fig. (3). Relationship between $\ln$ (reproductive success) and $\ln$ (egg production). (a) Plot where 1992-1995 data (in red) are included. When four data are included, two circles are observed, (b) According to these two circles, I separated the data into two periods, i.e., 1951-1972 (black) and 1974-1995 (red) and adopted the regression lines for each period. Thick blue regression line indicates the regression line estimated for the data from 1951 to 1995 . Here, $x$ and $y$ denote the $\ln$ (egg production) and $\ln$ (reproductive success), respectively. (c) For the latter group, the four year from 1988 to 1991 was removed and adapted a regression line. The slope was -0.236 and $p$ value 0.067 is statistically significant under the $10 \%$ significant level. The regression line for all data except from 1988 to 1991 , the slope was not different from zero and density-dependent effect cannot be detected.

the data from these four anomalous years is largely what determines the significance of the relationship between $\ln$ (reproductive successes) and $\ln$ (egg production). I consider this a worrisome finding.

\section{Results with 1992-1995 Data Included}

Wada and Jacobson [3] did not use their 1992-1995 data, because they considered the estimate values to be unreliable. Fig. (3a) plots the full data set, with the 1992-1995 data in red. With the 1992-1995 data included, the impression conveyed by Fig. (3a) is quite different. The existence of two separate relationships, for the favorable and unfavorable regimes, respectively (as Wada and Jacobson [3]), seems less apparent. Instead, two circles emerged (Fig. 3a), the first and the second circles appear in the lower and the higher levels of egg production, respectively. The first circle is con- structed by 1951-1972 data and the second one is constructed by 1974-1995 data. When we adapt the regression lines to these two circles, from 1951-1972 (closed black) and from 1974-1995 (closed red), the regressions for each period respectively yield significantly negative slopes of -0.902 $(p=0.009)$ and $-0.718(p=0.005)$ (Fig. 3b). The thick blue line shows the regression for the entire data set (1951-1995), for which the slope of -0.231 is statistically negative $(p=0.015)$.

Fig. (3c) illustrates how the regressions for the group from 1974 to 1995 change when the anomalous 1988-1991 data are excluded. The estimated slope of the regression line showed the shallower (relative to Fig. 3b) negative slope of $0.236(\mathrm{p}=0.067)$. This slope is also statistically negative under the $10 \%$ significant level. The regression slope for all the 1951-1995 data excluding 1988-1991 data, is only -0.036 $(\mathrm{p}=0.665)$ and is not statistically negative. 


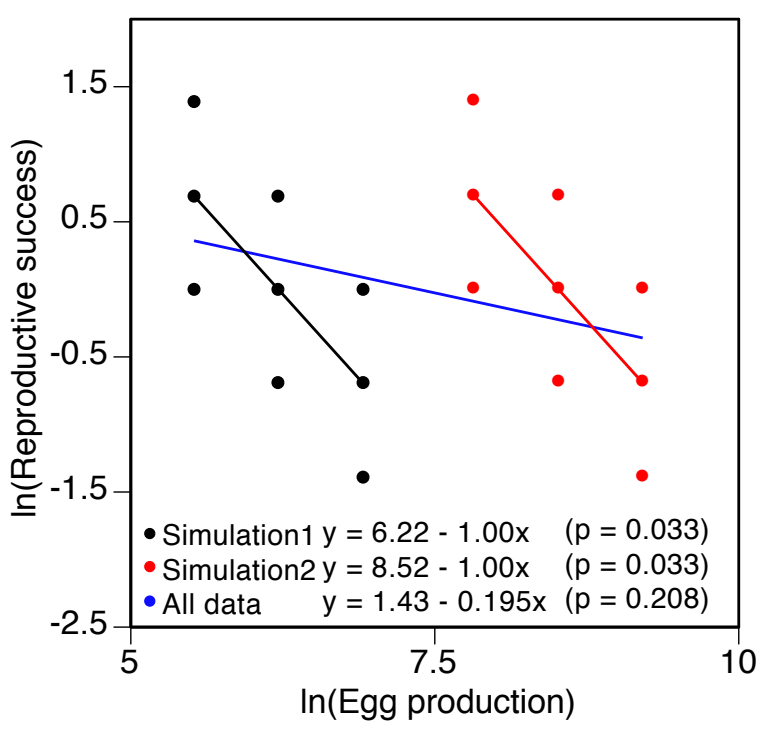

Fig. (4). Results of the simulations of which values are shown in Table 1. Black and red lines indicate the regression lines adapted for simulation 1 and simulation 2, respectively. Those slopes show significantly negative. However, the thick blue line that shows the regression line estimated for all data of simulation 1 and simulation 2 , is not significantly negative and statistically deferent from zero. This simulation indicates that reproductive success tend to produce the false negative trend when observation errors are incorporated and length of data is short. However, the length of data is long and level of egg production is quite deferent, those apparent decreasing trends are not detected. Here, $x$ and $y$ denote the $\ln$ (egg production) and $\ln$ (reproductive success), respectively.

The results obtained here indicated that the shape of the plot of $\ln$ (reproductive successes) against $\ln$ (egg production) is quite deferent when the data from 1991 to 1995 are included. In this case, it seems intuitively better to separate the data into two groups depending on the circle emerged rather than separating favorable and unfavorable regimes that Wada and Jacobson did [3]. When we adopt the regression lines for each group, the estimated values of the slopes for the shorter periods were always more steeper than the case when the regression line was adopted for longer data, regardless whether or not the anomalous four years are excluded.

When anomalous four years are included, the slopes for two group are -0.902 and -0.718 , respectively, whereas for all data, the slope is only -0.231 . When anomalous four years are excluded, the slopes for two circles are -0.902 and -0.236 , respectively, whereas for all data the slope is only -0.036 . If the density-dependent effect is truly exist, the results will be opposite, because the power to detect the density-dependent effect will increase as the length of data is longer. Wada and Jacobson (1998) noted that reproductive success in sardines responds rapidly to environmental changes, but that they can only be measured using long-term data series exhibiting large (1000-fold) changes in abundance [5]. However, the results obtained here are diametrically opposed to those noted by Wada and Jacobson (1998). That is, the slope of the regression line shows the steeper decreasing trend for the short period of data without the large change in abundance (egg production), however, the slope shows the shallower for the long period of data with large change in abundance. The minimum and maximum egg pro- ductions are $3 * 10^{12}$ in 1970 and $7,310 * 10^{12}$ in 1988 , then the latter is more than 2,400 times larger than the former.

Why did the phenomenon occur? I try to explain the mechanism hidden in the above phenomenon using simple simulation study in the next section.

\section{Simulation Analysis}

In Fig. (3b and $\mathbf{c}$ ), I showed that when we plot the $\ln$ (reproductive success) versus $\ln ($ egg production), the slope of the regression line shows the steeper decreasing trend for the short period of data without the large change in abundance (egg production), however, the slope shows the shallower for the long period of data with large change in abundance. These phenomena can be reproduced with simple and deterministic numerical simulations (Table 1).

In the simulations, the true reproductive success can be, of cause, obtained when $\mathrm{R}$ and egg production have no observation errors. Even when $\mathrm{R}$ and egg production have observation errors, if the observation errors in $\mathrm{R}$ and egg production are equally underestimated 0.5 -fold or overestimated 2.0-fold, the calculated reproductive success is coincided with the true value. For the other cases, however, the calculated reproductive successes have a bias of either underestimation or overestimation. When egg production was underestimated, the observed reproductive success was always overestimated, and vice-versa. Therefore, the graph in which the values for $\ln$ (reproductive success) are plotted against $\ln$ (egg production) shows clear decreasing trends (Fig. 4 left). The slope of the regression line was $-1.000(p=0.033$ $<0.05$ ), and the negative slope was statistically significant. This was the mechanism by which the slope of the regression line of $\ln$ (reproductive success) against $\ln$ (egg production) showed a false negative slope in response to observation errors, even for a short series of data points (Fig. 4 left).

The simulation 2 was conducted using the right hand column in Table 1. For the 9 data in simulation 2, the slope was the same as that for simulation 1 . However, the slope of the regression line adopted for all data in simulations 1 and 2 was $-0.195(\mathrm{p}=0.208>0.05)$ and $95 \%$ confident limit was $(-0.509,0.120)$. Therefore, in this case, the negative slope of the regression lines for each group is statistically significant; however, the slope of the regression line for all data was not significantly negative and did not differ significantly from zero.

These simple deterministic numerical simulations indicate that, even in a short series of data, a false decreasing trend in the regression line of $\ln$ (reproductive success) against $\ln$ (egg production) can emerge in response to observation errors. However, when long-term series of data with large changes in $\mathrm{R}$ and egg production such as regime shift are considered, the significantly negative but false slope does not detect. Consequently, these simulations suggest how apparently decreasing trends were be falsely detected, even using data collected over short periods, and also that data collected over long periods showing large changes in $\mathrm{R}$ and egg production can circumvent this problem.

\section{Regression Analysis of $\ln (\mathrm{R})$ Against In (Egg Production)}

The results derived above show that the reproductive success is potentially a flawed measure of the density- 

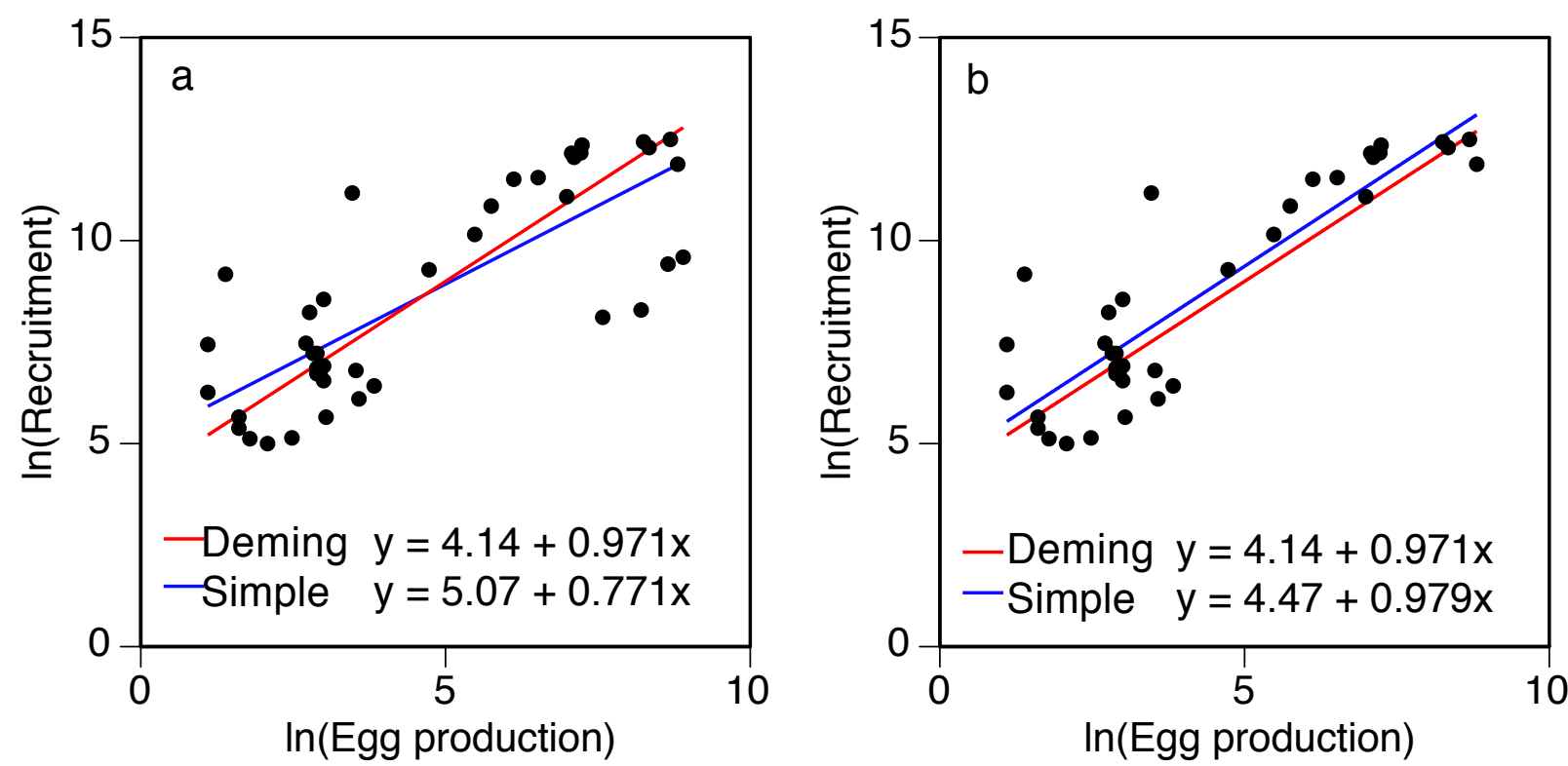

Fig. (5). Relationship between $\ln (\mathrm{R})$ and $\ln ($ egg production). (a) Regression line estimated for $\ln (\mathrm{R})$ against $\ln ($ egg production) for all data from 1951 to 1991. Blue and red indicate the regression line estimated by simple regression and the Deming regression, respectively. (b) Blue line indicates the regression line estimated by simple regression for $\ln (\mathrm{R})$ against $\ln ($ egg production) when the anomalous four years are removed. The red lines in Fig. (5a) and Fig. (5b) are same. Here, $x$ and $y$ denote the $\ln$ (egg production) and $\ln ($ reproductive success), respectively.

dependent effect of stock-recruitment relationship because it is so strongly influenced by questionable observations. Instead, it appears preferable to use the regression analysis of $\ln (\mathrm{R})$ versus $\ln ($ egg production). When simple regression analysis was employed for the data from 1951-1991, the slope of the regression line $(b=0.771)$ was significantly less than 1.0 , because which $95 \%$ confidence limit was $(0.557$, 0.965 ), whereas the slope of the regression line for the data excluding the four anomalous years $(b=0.979)$ did not differ significantly from 1.0 , because which $95 \%$ confidence limit was $(0.801,1.157)$ (Table 2, Fig. 5).

When Deming regression was applied to the data from 1951 to 1991 , the slopes of the regression lines $(b=0.971)$ did not differ significantly from 1.0, without excluding the four anomalous years' data, because which $95 \%$ confidence limit was $(0.866,1.099)$. The $95 \%$ confidence limit of Deming regression was much smaller than that of simple regression indicates that Deming regression is more robust method to estimate the regression line for noisy data. The regression line estimates derived from the Deming regression applied to the data including four anomalous years and from the simple regression using excluding the four anomalous years' data coincided well each other (Fig. 5b).

When we employ the Deming regression approach, the ratios of the variances in recruitment and egg production are necessary. In this study, we arbitrarily give the ratios as 1.0; however, even if we increase the variance ratio value to exceed $1.0,2.0, \ldots, 5.0$, the results are not so much different. When the ratio is set at 5.0, the slope estimated was 0.824 and $95 \%$ confidence limits of the slope was $(0.699,1.022)$. Therefore, the estimated slope is also recognized as statistically different from unity. When the slope of the regression line does not differ from 1.0, we can use the following stockrecruitment relationship model: $\ln (\mathrm{R})=\ln (\mathrm{a})+\ln ($ egg production $)$

or

$\mathrm{R}=\mathrm{a} \times$ egg production

The ability to describe the stock recruitment relationship for the Japanese sardine using the proportional model calls into question the claim of there being a density-dependent effect.

\section{DISCUSSION}

On the treatment of the data from 1988-1991

As shown in Fig. (1c), the reproductive successes in 1988-1991 were extremely low. The analytical treatment of these four years' data is very important because, depending upon whether they are included or excluded, the consequent results differ substantially. When a simple regression analysis was applied to the data series, with the 1988-1991 anomalous year data removed, a density-dependent effect was not detected for either the $\ln$ (reproductive successes) versus $\ln$ (egg production) or the $\ln (\mathrm{R})$ versus $\ln$ (egg production) regressions. If those four years' data were not removed, a density-dependent effect was detected in both regression analyses. Therefore, when we analyze the Japanese sardine data and discuss the density-dependent effect in stockrecruitment relationship, at least, we should calculate both the cases, four year's data from 1988 to1991, are included or excluded, and should discuss the diametrically opposed two results. Wada and Jacobson, however, analyzed only the latter case and insisted that the density-dependent effect typically exist. The assertion proposed in this paper is that when we discuss the density-dependent effect in stockrecruitment relationship for Japanese sardine, the former case, four year's data from 1988-1991 are excluded, is much more rational based on the many facts shown in this paper. 
Environmental changes could yield a sudden and dramatic decrease in recruitment over a period of four years. Sakuramoto et al. [11] showed that the Arctic Oscillation, the Pacific Decadal Oscillation, and the sea-surface temperature in the Kuroshio Extension areas were significantly associated with these drastic reductions. These are strong evidences that these four year's data are anomalous and should be excluded from the analysis when we discuss the densitydependent effect in stock-recruitment relationship using the simple regression analysis.

Based on an evaluation using Akaike's information criterion [15], Shimoyama et al. [16] noted that the recruitment data of Japanese sardine from 1988-1991 should be removed from the analysis of stock-recruitment relationship. This is consistent with there being large differences in the variance in egg production between the full data set and the same set from which the 1988-1991 data are removed. The variance in egg production estimated with Deming regression was 2.040 for all data from 1951 to $1995(n=45)$; however, it decreased to only 1.428 for the data that 4-year was removed $(n=41)$, where $n$ indicates the number of years over which each data set was collected. By removing only four years' data from the total series, which is corresponding to $8 \%$ reduction of the total number of data, the variances decreased by $70 \%$ relative to that for the full data set, which is corresponding to $30 \%$ reduction of the total variance. This further supports the assertion that the data for the four years from 1988 to 1991 should be considered as outliers and should be removed from analysis.

When we used Deming regression to model the $\ln (\mathrm{R})$ versus $\ln ($ egg production) relationship, the regression slope did not differ from 1.0, without removing the 4 years. Because Deming regression can treat the case when not only a dependent variable but also independent variables have observed errors. The estimated slope by simple regression analysis adapted for the data removed four years from 1988 to 1991 and that estimated by Deming regression applied for all data were coincided extremely well. The $95 \%$ confidence limit of Deming regression was much smaller than that of simple regression analysis. These would be evidences of the validity removing the four years from 1988 to 1991 as the anomalous years when we use the simple regression analysis, and the validity of the method of Deming regressing that can estimate a robust estimation, especially in the case when the independent valuable has observation errors. Therefore, the reliability of the result that the density-dependent effect in stock-recruitment relationship of Japanese sardine was not detected in the data would be high.

Overall, this study shows that using reproductive successes to detect density-dependent effects on stock-recruitment relationship offers little utility. As the simple deterministic simulations demonstrated, even when the period of data collection is short (for instance, even within a regime), reproductive successes falsely yields a decreasing trend in stock-recruitment relationship as a consequence of due erroneous observations. However, when the data series is longer and includes two regimes (and the level of egg production is differs substantially between those two periods), the false decreasing trend does not detected. This outcome indicates that when stockrecruitment relationship data for the Japanese sardine are ana- lyzed using the simple regression analysis, the data from 1988-1991 should be basically excluded and the use of reproductive successes statistics avoided.

On a treatment of 1973 data in Fig. (3)

In Fig. (3), I adapted the regression line excluding the 1973 data, because it was difficult to judge whether it belongs to the first circle or the second circle. If the data belongs to the second circle, the regression lines and significant probabilities were $-0.813(p=0.0005)$ for the data from 1973 to 1995 , and $0.423(p=0.0038)$ for the data from 1973 to1987 and 1991to1995, respectively. As the high value of reproductive success in 1973 was incorporated into the second circle, both of the slopes and significant probabilities were much steeper and extremely lower. However, the qualitative feature was not changed from those that I noted in Fig. (3) and Fig. (4).

On the contrary, when the 1973 data was incorporated into the first circle, the slope and the significant probability changed as $-0.627(p=0.117)$ for the data from 1951-1973. However, the slope was steeper than that estimated for all data. Therefore, the same logic that I explained in Fig. (3) and Fig. (4) can also be adapted for this case.

\section{DO TWO CARRYING CAPACITIES EXIST BE- TWEEN DIFFERENT REGIME TYPES?}

Yatsu et al. [8] analyzed stock-recruitment relationship data for the Japanese sardine from 1951 to 2000 that incorporated the effects of various environmental factors and derived the same results of Wada and Jacobson [3], i.e., Yatsu et al. [8] concluded that environmental conditions were favorable for Japanese sardine during 1969-87 and unfavorable during 1951-67 and after 1988. Further they noted that the transition from a favorable to an unfavorable regime generally starts with the stock at relatively high biomass. Both instantaneous surplus production rate and log recruitment residual (differences between data and estimated values calculated by simple Ricker model) fall rapidly when environmental conditions change to a less favorable condition because carrying capacity $(K)$ shifts to a lower level and stock biomass may initially exceed the new level of $K$. As biomass declines, density-dependent effects are reduced, both instantaneous surplus production rate and log recruitment residual increase toward new levels.

However, I strongly doubt that these carrying capacities differ substantially between the regime types. As shown in the present paper, the analyses of Yatsu et al. [8] exhibited the same mistake that Wada and Jacobson [3] made, i.e., they did not exclude the 1988-1991 data (only four of the 50 years' data). If they had done so, the $\ln$ (reproductive successes) versus $\ln ($ egg production) regression slope for 1951 to 2000 data would have been slightly negative $(-0.121)$ but not significantly so $(\mathrm{p}=0.924)$, consistent with stock recruitment relationship neither being density-dependent effect nor being two different carrying capacities, leaving the proportional model as being more reasonable for the Japanese sardine, even for long-term series of data from 1951-2000.

Sakuramoto and Suzuki [17] discussed in detail the effect of observation and/or process error, which mainly derived from environmental factors, in R and/or SSB in the selection of an stock-recruitment relationship model. 
They showed that when the true model (i.e., the one assumed in the simulation) is the proportional model, there is a high probability that process and/or observation errors will result in the estimated stock-recruitment relationship model being mis-identified as either the Ricker or the Beverton and Holt model. In contrast, when the true model was the Ricker or the Beverton and Holt model, process and/or observation errors seldom resulted in the proportional model being selected. This outcome suggests that if the proportional model is indeed identified as fitting actual stock-recruitment relationship data, then there is a high probability that the relationship between $\mathrm{R}$ and egg production could instead be fit by the proportional model.

\section{UNDERESTIMATION OF THE EFFECTS OF ENVI- RONMENTAL FACTORS}

Yatsu et al. [8] and Ishida et al. [18] adapted extended Ricker models that incorporate environmental factors and proposed extended stock-recruitment relationship models for the Japanese sardine. However, as Sakuramoto and Suzuki [17] noted, the application of such approaches will result in serious mistakes when the true stock-recruitment relationship model lacks a density-dependent effect. Because Yatsu et al. [8] and Ishida et al. [18] assumed a priori that a densitydependence effect existed in the relationship between $\mathrm{R}$ and egg production. However, if no density-dependent effect exists in the original SRR and the density-dependent effect is erroneously estimated in response to observation error or the density-dependent effect appears in response to the process error (environmental factors), the extended Ricker model that has independent variables related to environmental factors is used for estimating recruitments, the parameters related to the effect of environmental factors will be underestimated, because some parts of the environmental effect will be counted as the density dependent effect that is assumed intrinsically existing in the Ricker model.

\section{VALIDITY OF THE CONCEPT OF MSY AS THE TARGET OF FISHERIES MANAGEMENT}

If the proportional model reasonably approximates the true stock-recruitment relationship model, concepts of resource management for the Japanese sardine should be revised because the optimal stock level (from a biological perspective) will not exist at any stock level less than the carrying capacity. In this context, a carrying capacity implies the maximum stock size that have ever been observed. In other words, the biologically optimal stock level can only be achieved when there is no harvesting. However, it is not my intention to assert that harvesting should be prohibited: I believe that marine biological resources should be utilized by people in a reasonable manner. Rather, my point is that the management of such resources will fail if harvest strategies are based on an incorrect concept of density-dependent effects in stock-recruitment relationship, leading to erroneous management targets such as an overall MSY, or an MSY for two regimes as Wada and Jacobson [3] and Yatsu et al. [8] insisted.

\section{BIAS EXCLUDED IN THIS ANALYSIS}

This study indicates that density-dependent effect in the stock-recruitment relationship never be detected in the Japa- nese sardine of Wada and Jacobson [3] data. However, it bears noting that Wada and Jacobson's [3] values for R and egg production are also both estimated values. If there were bias in these values, such that the degree of over-estimation of $\mathrm{R}$ increased as the estimate of egg production increased, then the mechanism suggested here for how stockrecruitment relationship is affected might not be valid. In latter this case, one would be well served to consider other interpretations concerning the density-dependent effect between $\mathrm{R}$ and egg production, or that further evaluation of the mechanisms yielding population fluctuation is required.

\section{CONFLICT OF INTEREST}

The authors confirm that this article content has no conflicts of interest.

\section{ACKNOELWDGEMENTS}

I thank two anonymous reviewers for their many useful suggestions that led to improvements in the manuscript. I also thank Editage, a division of Cactus Communications for their assistance with the translation and editing of this manuscript.

\section{REFERENCES}

[1] Ricker WE. Stock and recruitment. J Fish Res Board Can 1954; 11: 559-623.

[2] Beverton RJH, Holt SJ. On the dynamics of exploited fish populations. Fish Invest Ser Mar Fish GB Minist Aguric Fish Food 1957; 19:1-533.

[3] Wada T, Jacobson LD. Regimes and stock-recruitment relationships in Japanese sardine (Sardinops melanostictus) 1951-1995. Can J Fish Aquat Sci 1998; 55: 2455-63.

[4] Jacobson LD, MacCall AD. Stock-recruitment models for Pacific sardine (Sardinops sagax). Can J Fish Aquat Sci 1995; 52: 566-77.

[5] Hilborn R, Walters CJ. Quantitative fisheries stock assessment: choice dynamics and uncertainty. New York; Chapman and Hall 1992.

[6] Cergole MC, Saccardo SA, Rossi-Wongtschowski CLDB. Fluctuations in the spawning stock biomass and recruitment of the Brazilian sardine (Sardinella brasiliensis) 1977-1997. Bras J Oceanogr 2002; 50: 13-26.

[7] Tanaka E. A method for estimating dynamics of carrying capacity using time series of stock and recruitment. Fish Sci 2003; 69: 67786.

[8] Yatsu A, Watanabe T, Ishida M, Sugisaki H, Jacobson LD. Environmental effects on recruitment and productivity of Japanese sardine Sardinops melanostictus and chub mackerel Scomber japonicus with recommendations for management. Fish Oceanogr 2005; 14: 263-78.

[9] Munch SB, Kottas A. A Bayesian modeling approach for determining productivity regimes and their characteristics. Ecol App 2009; 19: 527-37.

[10] McClatchie S, Goericke R, Auad G, Hill K. Re-assessment of the stock-recruit and temperature: recruit relationships for Pacific sardine (Sardinops sagax). Can J Fish Aquat Sci 2010; 67: 1782-90.

[11] Sakuramoto K, Shimoyama S, Suzuki N. Relationships between environmental conditions and fluctuations in the recruitment of Japanese sardine Sardinops melanostictus in the northwestern Pacific. Bull Jpn Soc Fish Oceanogr 2010; 74: 88-97.

[12] Stöckl D, Dewitte K, Thienpont LM. Validity of linear regression in method comparison studies: is it limited by the statistical model or the quality of the analytical input data? Clin Chem 1998; 44: 2340-6.

[13] Martin RF. General Deming regression for estimating systematic bias and its confidence interval in method-comparison studies. Clin Chem 2000; 46:100-4.

[14] Aoki S. Parameter estimation of a regression line by the Deming regression method. updated Available from http://aoki2.si.gunma- 
u.ac.jp/R/Deminghtml [accessed $3^{\text {rd }}$ Sept 2009, cited 23 rd April 2012].

[15] Akaike H. Likelihood of a model and information criteria. J Eco 1981; 16: 3-14.

[16] Shimoyama S, Sakuramoto K, Suzuki N. Proposal for stockrecruitment relationship for Japanese sardine Sardinops melanostictus in the North-western Pacific. Fish Sci 2007; 73:1035-41.
[17] Sakuramoto K, Suzuki N. Effects of process and/or observation errors on the stock-recruitment curve and the validity of the proportional model as a stock: recruitment relationship. Fish Sci 2012; 78: 41-54.

[18] Ishida Y, Funamoto T, Honda S, Yabuki K, Nishida H, Watanabe C. Management of declining Japanese sardine chub mackerel and walleye pollock fisheries in Japan. Fish Res 2009; 100: 68-77.

Received: September 05, 2012

(C) Kazumi Sakuramoto; Licensee Bentham Open.

This is an open access article licensed under the terms of the Creative Commons Attribution Non-Commercial License (http://creativecommons.org/licenses/ by-nc/3.0/) which permits unrestricted, non-commercial use, distribution and reproduction in any medium, provided the work is properly cited. 